Classification

Physics Abstracts

$61.50 \mathrm{C}-74.70 \mathrm{~V}-75.50 \mathrm{M}$

\title{
Material processing in high static magnetic field. A review of an experimental study on levitation, phase separation, convection and texturation
}

\author{
E. Beaugnon, D. Bourgault, D. Braithwaite, P. de Rango, R. Perrier de la Bathie, A. \\ Sulpice and R. Tournier
}

Centre de Recherches sur les Très Basses Températures, CNRS, 25 Avenue des Martyrs, BP 166, F38042 Grenoble Cedex 09, France

(Received 18 June 1992, accepted in final form 22 July 1992)

\begin{abstract}
An inhomogeneous magnetic field exerts a force on magnetic materials and can be used either to compensate gravity, control convection in a magnetic liquid or modify the microstructure of a solidified multiphased system where the phases exhibit different magnetic susceptibilities. A homogeneous field may produce a preferred crystallographic alignment in systems which exhibit an anisotropy of their magnetic susceptibility near their melting point. We present an experimental study of those effects, demonstrating their impact on the elaboration of a wide range of materials.
\end{abstract}

\section{Introduction.}

Few studies have been made on the use of high static magnetic field during solidification as a way to control the microstructure of various materials [1-3]. We present experimental results on four aspects of the use of a magnetic field as a tool for material growth. We show that using the high fields which are now produced by experimental or commercial apparatus, many substances may be maintained in stable levitation. With a field strength far less than that required for levitation, one can control, either suppress or enhance, the thermal convection in an insulating or conducting liquid, providing its magnetic susceptibility varies with temperature. We discuss the impacts of such effects when applied to material growth.

We present new experimental results showing that both homogeneous and inhomogeneous fields applied during solidification deeply influence the microstructure of various systems. The magnetic force induced by an inhomogeneous field on a magnetic material can separate the different phases that appear during solidification of a multiphased system. A homogeneous magnetic field induces a preferred crystallographic orientation in anisotropic materials. This orientation effect has been studied with congruent melting alloys and during the peritectic reaction of $\mathrm{YBa}_{2} \mathrm{Cu}_{3} \mathrm{O}_{7}$ and reveals an irreversible behavior of the liquid state since we observe that the quality of the alignment depends on the thermal history of the liquid. In the case 
of $\mathrm{YBa}_{2} \mathrm{Cu}_{3} \mathrm{O}_{7}$, improved superconducting properties are evidenced, due to crystallographic alignment of grains.

\section{Levitation.}

A magnetic body of moment $M$ placed in a magnetic field gradient is submitted to a magnetic force whose expression $F=(M \cdot \nabla) B$ corresponds to a force which tends to drive the sample to a minimization of its magnetic energy. We have studied, both analytically and experimentally, the strength of this magnetic force and the conditions of stability of the levitation that can be obtained $[4,5]$.

In the case of isotropic paramagnetic and diamagnetic bodies which exhibit a linear variation of their magnetization versus field, the magnetic force per unit volume may be expressed as $F=-\left(\chi / 2 \mu_{0}\right)$ grad $\left(B^{2}\right)$, where $\chi$ is the magnetic susceptibility in S.I. units. In field geometries such as those obtained with a vertical cylindrical coil, this magnetic force can be exerted upward to counterbalance the gravity and maintain material in levitation, providing the susceptibility and the applied field gradient are high enough. In fact, even the lowest susceptibilities of diamagnetic materials allow levitation since apparatus are nowadays able to produce very high field strength.

In the high field produced by the hybrid coil of the Service National des Champs Intenses in Grenoble, we have observed the levitation of various liquid and solid diamagnetic substances. Samples were placed in a region of high inhomogeneity of the field, a few centimeters above the center of the coil. In this position, the magnetic force is exerted upward and can be adjusted to exactly compensate gravity. The levitation of the samples has been observed from the top plate of the magnet and by an in situ optical device. Results, which are summarized in Table I, confirm that even low susceptibility materials can be levitated. The case of organic material is of prime interest, since their magnetic specific diamagnetic susceptibility - which varies only a little from an element to another - allows microgravity elaboration.

Table I. - Levitation of diamagnetic solids and liquids in the high field hybrid magnet of the S.N.C.I. (Grenoble). In each case, we have measured the magnetic field at the center of the coil and the field - field gradient product applied on the samples. Except for liquids where the wetting effect on the crucible should be taken into account, the observed values agree with the expected ones calculated from the known susceptibility of these elements.

\begin{tabular}{|l|c|c|}
\hline & $\begin{array}{c}\text { maximum field in the } \\
\text { center of the coil }(\mathrm{T})\end{array}$ & $\begin{array}{c}\text { field }- \text { field gradient } \\
B \mathrm{~d} B / \mathrm{d} z\left(\mathrm{~T}^{2} / \mathrm{cm}\right)\end{array}$ \\
\hline Water & 27 & 30 \\
\hline Ethanol & 21 & 16 \\
\hline Acetone & 22 & 20 \\
\hline Bismuth & 15.9 & 7.3 \\
\hline Antimony & 18.8 & 12 \\
\hline Wood & 21.5 & 17 \\
\hline Plastic & 22.3 & 20 \\
\hline
\end{tabular}


The stability of the levitation is expected for diamagnetic substances but it has not been verified in our experimental apparatus. We have therefore performed a specific study of this effect, taking advantage of the high specific susceptibility of graphite and using a lower field magnet with a larger bore $(12 \mathrm{~cm})$ for easier experimentation. We have observed that for specific conditions of the field strength, stability of the levitation was obtained : any deviation of the sample makes it freely oscillate around its rest position. The calculated field map of the magnet confirms these observations. In figures la to $1 c$, we represent the specific energy of a sample of graphite as a function of its position in the coil. This energy is the sum of the specific gravitational energy gz and the specific magnetic energy $\left(\chi / 2 \mu_{0}\right) B^{2}$. a local minimum of this energy indicates a stable position for levitation. One can see that below a certain field, the magnetic force will not be strong enough to obtain levitation (Fig. 1a). Between two limits, the magnetic force is strong enough to compensate gravity and a local minimum of the energy allows the stability of the levitation on the axis of the coil. For fields above the upper limit, vertically stable levitation is still obtained but the radial forces drive the sample away from the center axis until it comes in contact with the bore tube of the magnet. This behavior has been experimentally confirmed with a sample of polycrystalline graphite. We have also studied the levitation of a single crystal of graphite : due to the large anisotropy of the susceptibility, we could not reach fields high enough to obtain levitation. The susceptibility along the $a b$ plane of the hexagonal structure is $-0.5 \times 10^{-6} \mathrm{emu} / \mathrm{g}$ while it is about $-22 \times 10^{-6}$ along the $c$ axis. The single crystal tends to align with the direction of major algebraic susceptibility $(\chi a b)$ parallel to the field. The field required to obtain levitation is then much higher since this susceptibility is about 15 times smaller than the susceptibillity of a polycrystalline sample.

These results open the way to a new field of crystal growth in low gravity. Although it can also applied to paramagnetic material, this technique is more relevant in the case of diamagnetic substances since in addition to low gravity, contactless elaboration due to the stability of levitation could help to prevent contamination and reactivity from a crucible.

\section{Convection.}

It is well known that variation of fluid density with temperature leads to buoyancy driven convection. As a parallel, variation of the magnetic force on a paramagnetic liquid, due to Curie susceptibility, leads to a magnetically driven convection [6], this effect being independent of the electrical conductivity of the liquid. We have studied the magnetic convection of a gadolinium nitrate aqueous solution in a cylindrical vertical convection cell [7]. One of the plates of the cell was cooled by water while the other was heated by a resistive wire. The convection cell was placed in the room temperature bore of a vertical superconducting coil, either in the region of upward or downward force (Fig. 2). The applied thermal gradient was parallel to the magnetic force, being either directed upward or downward. The temperature difference between the hot and cold plates was recorded as a function of the applied force and the electrical power applied to the hot plate. The values were normalized to the case where only pure conduction exists in the liquid, with no field and the hot plate being on the top, thus giving the Nusselt number $\mathrm{Nu}$ versus $\Delta T$.

Figures $3 \mathrm{a}$ to $3 \mathrm{c}$ correspond to different combinations of the directions of the magnetic force and the thermal gradient. In figure $3 \mathrm{a}$, no convection exists in zero field since the hot plate is on top of the cell. With the application of an upward magnetic force, thermal exchange is greatly enhanced : the cold parts of the liquid, submitted to a greater force than the hot parts, are driven to the hotter top plate expelling the hot parts downwards ; exchange of heat leads to a reversal of the temperatures, hence keeping up the flow. In figures $3 b$ and $3 c$, the hot 


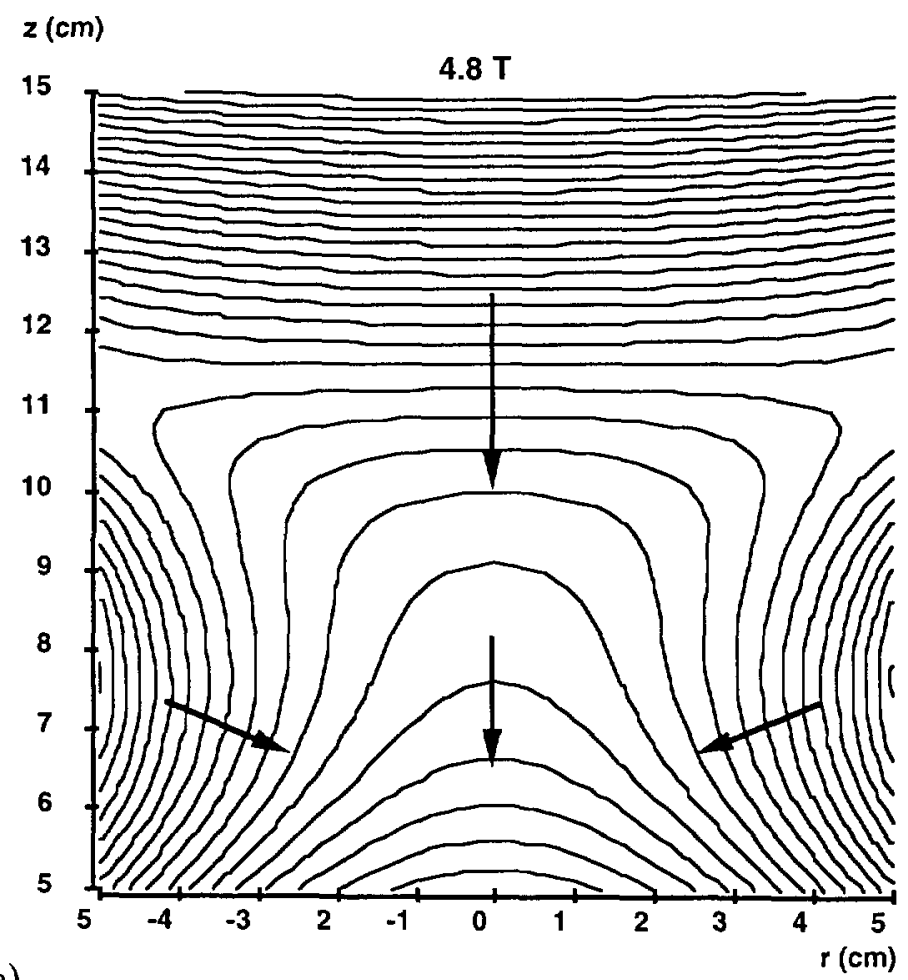

a)

Fig. 1. - Contour lines of the potential energy (gravitational and magnetic) of 1 gram of diamagnetic polycrystalline graphite as a function of its position in the field of a vertical superconducting coil. Each line joins the isovalues of this energy. Two lines are separated by $10^{-5} \mathrm{~J} / \mathrm{g}$. The descending slopes are indicated by arrows. The $z$ axis corresponds to the vertical distance from the center of the coil and the $r$ axis corresponds to the distance from: the axis of the coil. The 3 curve families were calculated for different values of the magnetic field at the center of the coil. The coil has a rectangular section from $z=-10 \mathrm{~cm}$ to $z=10 \mathrm{~cm}$ and $r=6.2 \mathrm{~cm}$ to $r=9.25 \mathrm{~cm}$. With 4.8 teslas (Fig. 1a), the magnetic force is not strong enough to obtain levitation : no minimum in the total energy can be seen. With 5.2 teslas (Fig. 1b), a minimum in the energy indicates the stability of the levitation at $r=0$ and $z=10$. With 6 teslas (Fig. 1c), the magnetic force expels the sample from the region of radial stability and drives it in contact with the magnet bore at $z=12 \mathrm{~cm}$.

plate is on the bottom of the cell, leading to buoyancy driven convection in zero field. One can see that depending on the orientation of the magnetic force, convection is either enhanced or suppressed.

In zero field, convection is governed by the dimensionless Rayleigh number $\mathrm{Ra}=g \beta \Delta T d^{3} / \alpha v$ where $\alpha$ if the thermal diffusivity, $v$ the kinematic viscosity, $\Delta T$ the thermal gradient, $d$ a characteristic length of the cell, $\beta$ the thermal expansion of the liquid and $g$ the acceleration due to gravity. Due to paramagnetism of the liquid and field gradient, the liquid is submitted to a force per unit mass :

$$
F_{\mathrm{m}}=\frac{\mu_{0} \chi}{\rho} H \frac{\mathrm{d} H}{\mathrm{~d} z}
$$

where $\chi$ and $\rho$ are the magnetic susceptibility and the volumic mass of the liquid and $H$ the 

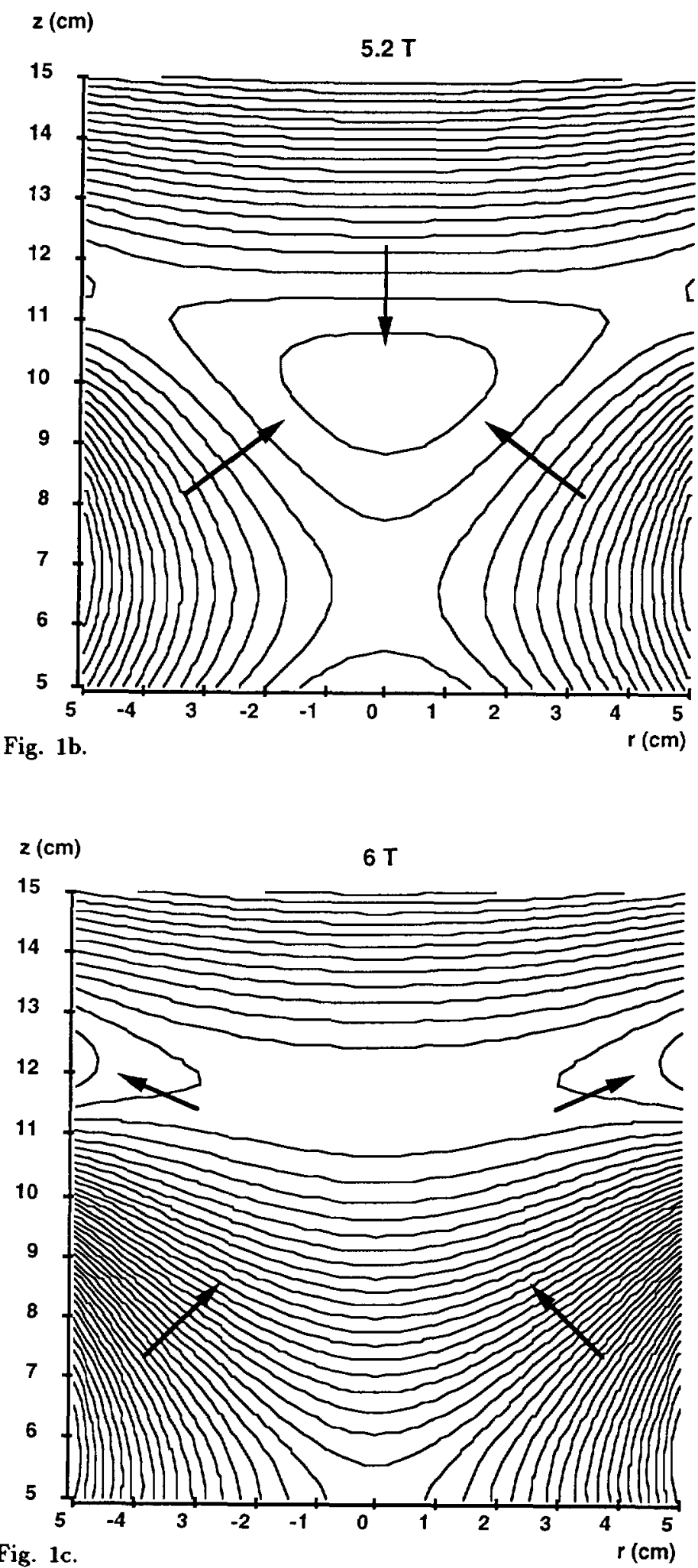


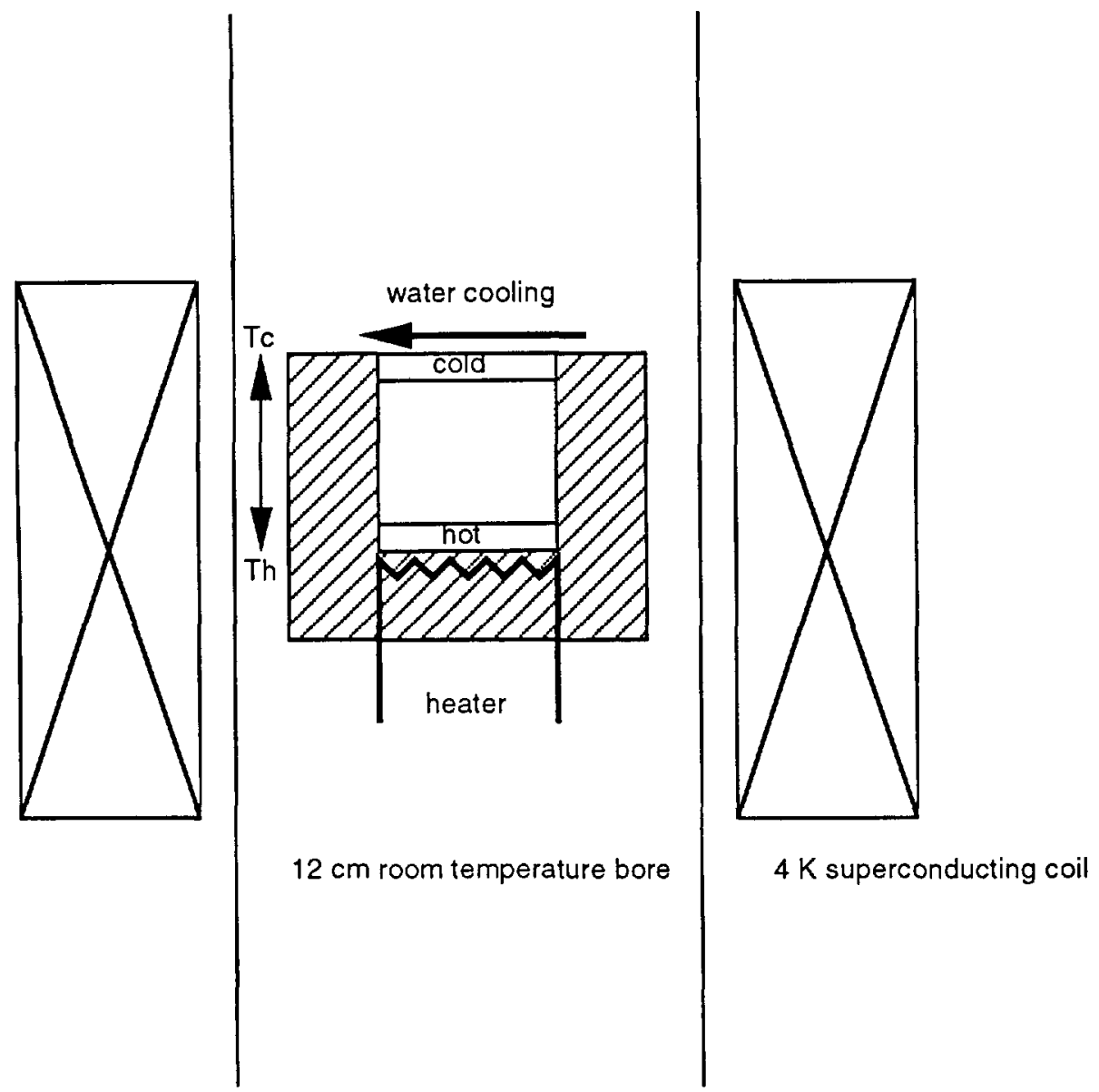

Fig. 2. - Design of the convection cell used to measure the thermal exchanges in a gadolinium nitrate solution placed in a region of inhomogeneous magnetic field. The cold and hot faces can be reversed as well as the sign of the magnetic force that depends on the position of cell.

applied field. This magnetic force contributes to an apparent variation of $g$. A second and more important effect is due to the variation of the force with the temperature since the susceptibility of the liquid obeys a Curie law $\chi=C / T$. Since the magnetic equivalent of $\beta$ is :

$$
\gamma=\frac{-1}{\chi} \frac{\mathrm{d} \chi}{\mathrm{d} T} \approx \frac{1}{T}
$$

we can define a magnetic Rayleigh number :

$$
\mathrm{Ra}_{\mathrm{m}}=\mathrm{Ra}\left(1+\frac{F_{\mathrm{m}}}{g}\left(1+\frac{\gamma}{\beta}\right)\right)
$$

If $\gamma=0$, the ratio $F_{\mathrm{m}} / g$ corresponds to the apparent variation of gravity, leading to zero Rayleigh number when $F_{\mathrm{m}}$ is equal to $-g$, i.e. in zero gravity due to levitation. The $\gamma / \beta$ ratio corresponds to the relative influence of buoyancy and magnetic convection and has a greater 

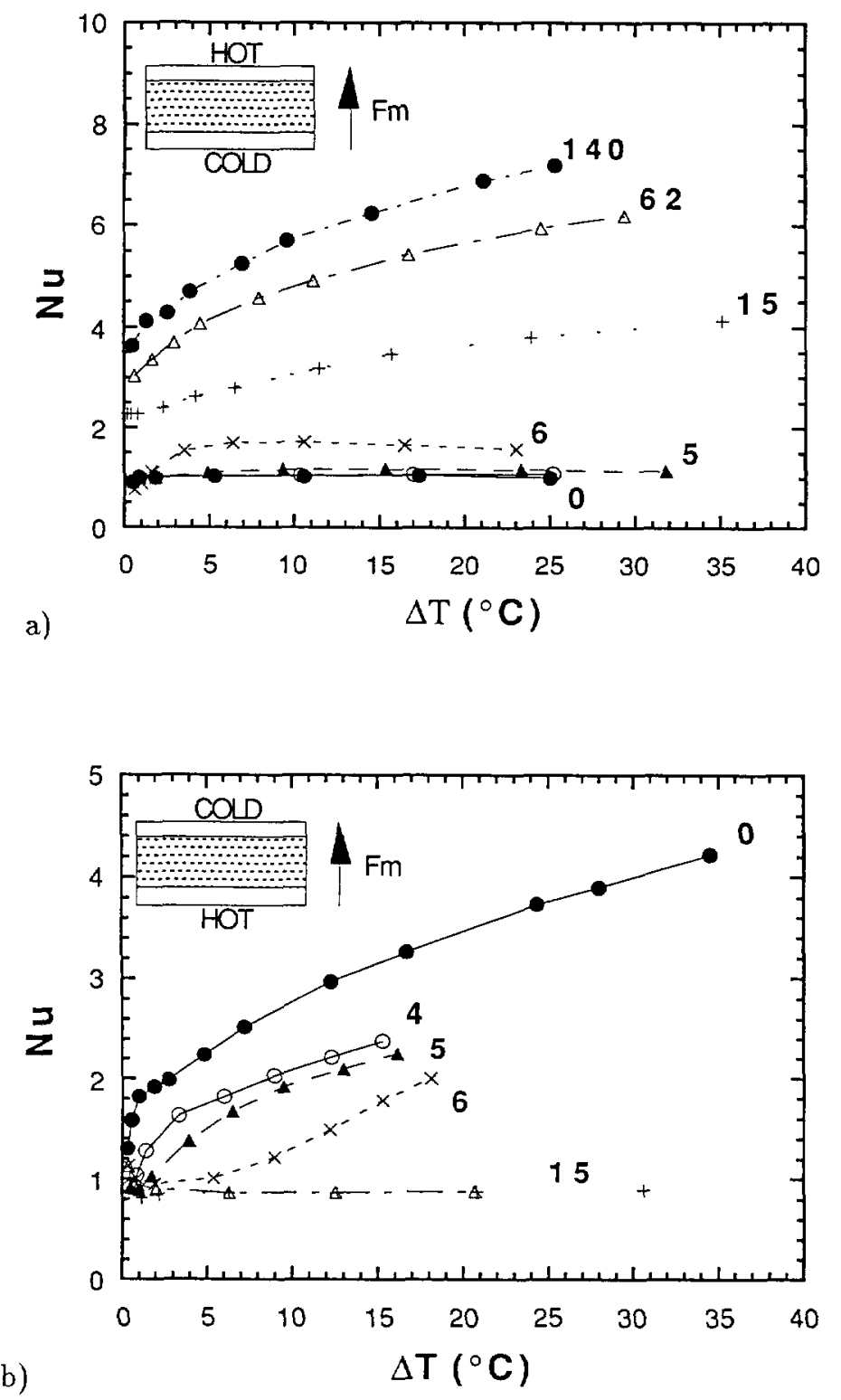

Fig. 3. - Plot of the Nusselt number versus thermal gradient in the convection cell for different orientations of the magnetic force and the thermal gradient. The different curves correspond to different amplitudes of the $B d B / \mathrm{d} z$ driving force expressed in $\mathrm{T}^{2} / \mathrm{m}$. a) No buoyancy driven convection exists since the hot plate is on the top of the cell. The upward magnetic force drives the cold parts of the fluid in contact with the hot plate and expels the hot parts downward in contact with the cold plate. The exchange of heat then maintains the flow. With a driving force of $140 \mathrm{~T}^{2} / \mathrm{m}$, the Nusselt number is multiplied by a factor 7 . b) The convection that exists in zero field is suppressed by the application of a moderate driving force (lower than $15 \mathrm{~T}^{2} / \mathrm{m}$ ) which maintains the cold parts on the top plate and stops the flow. c) The buoyancy driven convection is enhanced by the magnetic convection. 


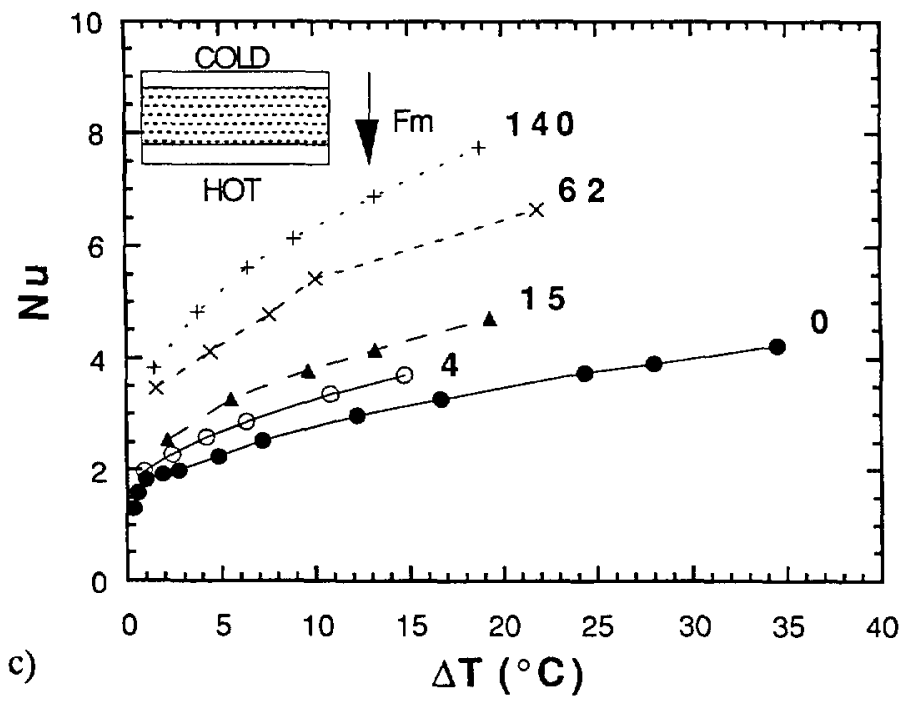

Fig. 3. - (continued)

effect on the value of $\mathrm{Ra}_{\mathrm{m}}$. With $4.33 \mathrm{~g}$ of gadolinium nitrate dissolved in $10 \mathrm{ml}$ of water, a $\mu_{0}^{2} H \mathrm{~d} H / \mathrm{d} z$ driving force of $15 \mathrm{~T}^{2} / \mathrm{m}$ is an upper limit to suppress convection while levitation should have required about $60 \mathrm{~T}^{2} / \mathrm{m}$. Convection is in this case governed by a high value of the ratio $\gamma / \beta=16$ at $T=20^{\circ} \mathrm{C}$, where $\beta$ is assumed to be the same than that of pure water $\left(\beta=2 \times 10^{-4} \mathrm{~K}^{-1}\right)$ and $\gamma=1 / 300 \mathrm{~K}^{-1}$

\section{Experimental apparatus for high static magnetic field elaboration.}

We have designed several special apparatuses which combine high magnetic field and high temperature to study the field effects on material elaboration. A typical assembly is described in figure 4. The magnetic field is provided by a superconducting coil, at liquid helium temperature, which gives a vertical access at room temperature in a 10 to $12 \mathrm{~cm}$ cylindrical bore. The maximum field, depending on the coil used, may be as high as $12 \mathrm{~T}$. In the room temperature bore, a vertical furnace cooled to room temperature by flowing water is inserted. Different technologies have been used to provide insulation and heating elements : a molybdenum wire embedded in a refractory mould, placed in vacuum, a Fe-Cr-Al alloy heating wire in air, wound on an alumina working tube with different inner atmosphere, or a $\mathrm{La}_{2} \mathrm{CrO}_{3}$ heating tube in air in which is placed a working tube in silica or alumina with a different inner atmosphere, the insulation of those different elements being performed by a thin wall refractory material. In each apparatus, attention had been paid to the use of non magnetic materials and current distribution parallel to the applied field to minimize mechanical coupling. In addition, d.c. power supplies were preferred to a.c. ones to suppress vibrations due to residual coupling.

In one of these apparatus, we have realized a high temperature magnetometer which records as a function of time and temperature the force exerted on the samples placed in a high field gradient (Fig. 5). The measuring cell, a precise weighing machine placed far from the maximum 


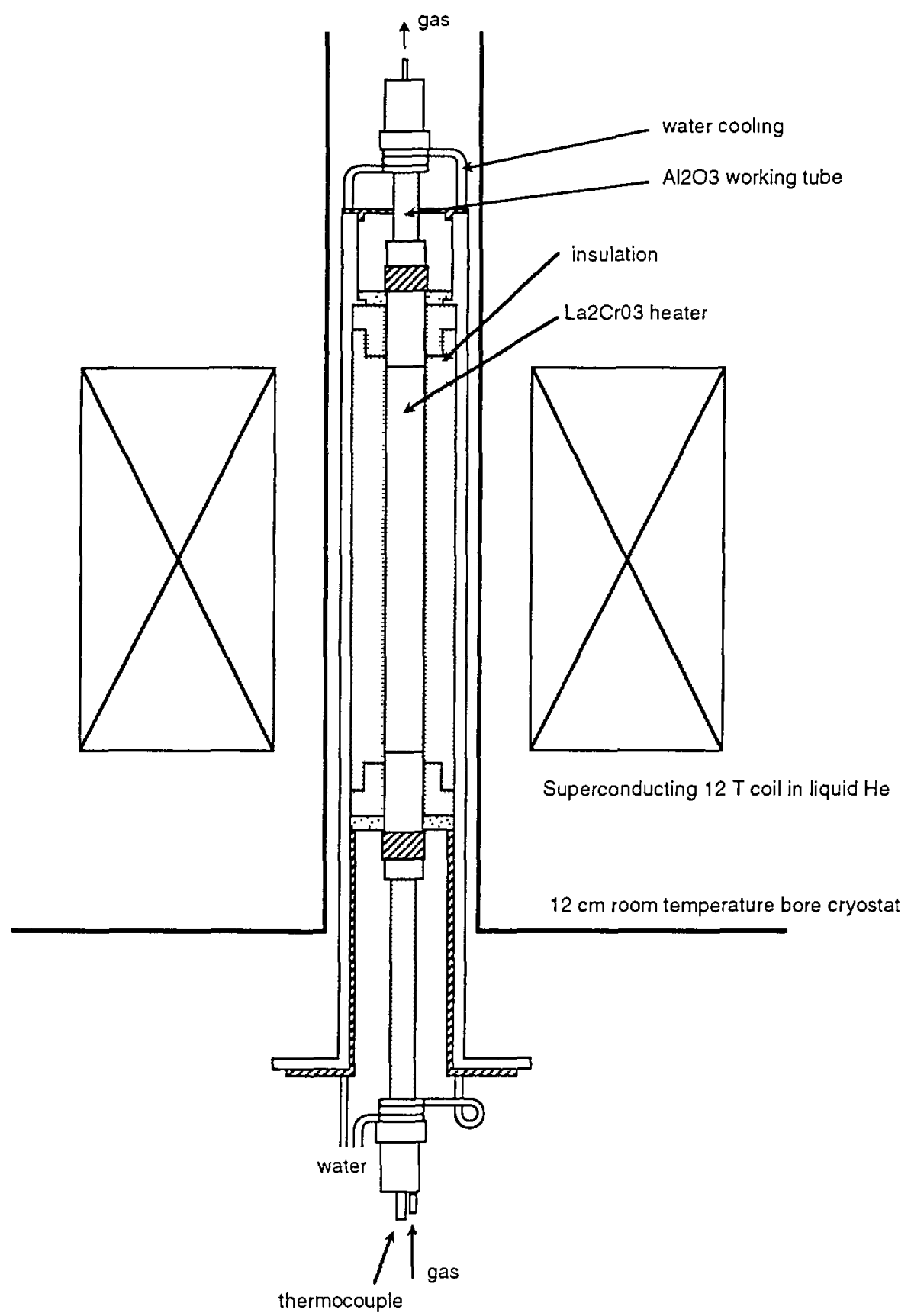

Fig. 4. - Design of an apparatus used to obtain high temperature in high field. A vertical water cooled furnace of small diameter is inserted in the room temperature bore of a superconducting coil. 
field, allows the measurement of very low magnetic susceptibilities with high accuracy in high field, the resolution being as low as a few $10^{-10} \mathrm{emu}$ in the best cascs.

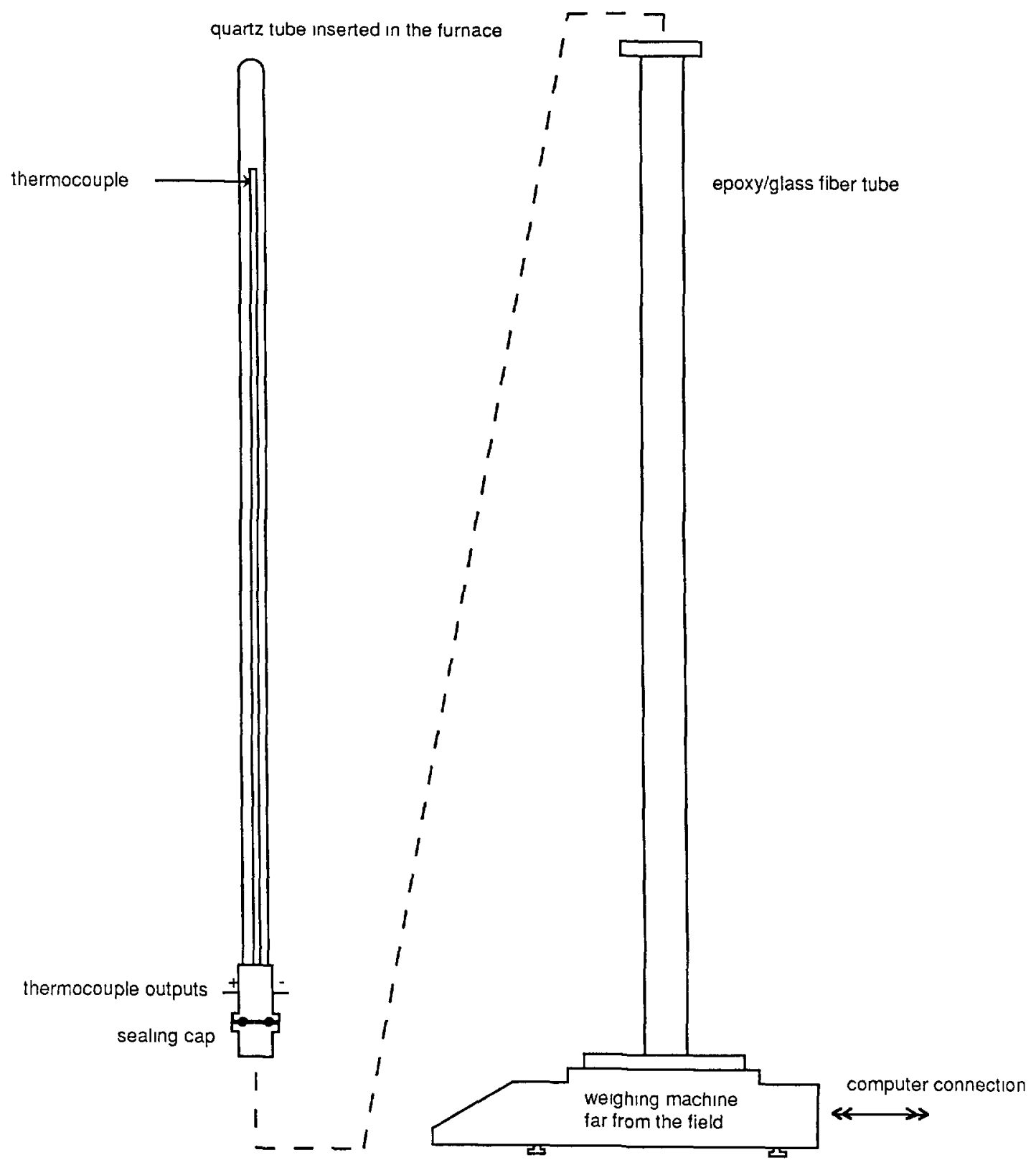

Fig. 5. - The force exerted on a magnetic sample under processing in the region of high field gradient is recorded by a precise weighing machine placed far from the field. 


\section{Phase separation.}

We have studied the solidification of an eutectic alloy $\mathrm{Nd}-\mathrm{Fe}$ while a strong magnetic force is applied. The phase diagram of this system, which has been recently studied by Landgraff et al. [8] is shown in figure 6. Although the eutectic stoechiometry should lead to a large scale homogeneous microstructure, non equilibrium phases are frequently observed, depending on the solidification process, leading to a mixture of $\mathrm{Nd}, \mathrm{Nd}_{2} \mathrm{Fe}_{17}$ and $\mathrm{Nd}_{5} \mathrm{Fe}_{17}[9,10]$.

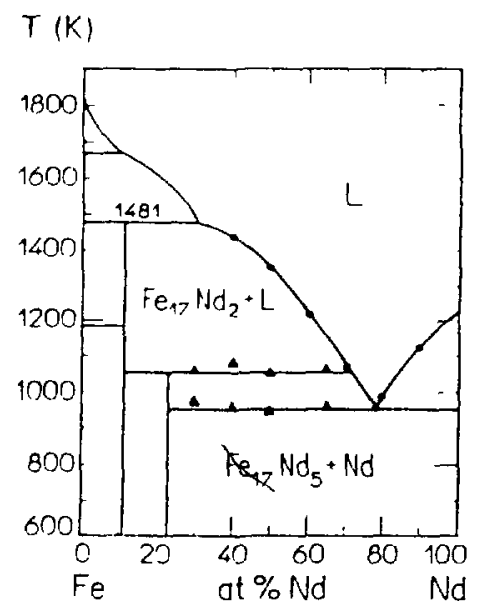

Fig. 6. - Phase diagram of the Nd-Fe system [8].

Samples of $\mathrm{Nd}$ and $\mathrm{Fe}$ at the eutectic composition were first prepared by cold-mold casting. The thermal treatment under magnetic field then applied to those samples is described in figure 7 . Two different values of the magnetic force were used depending on the temperature in order to optimize the recording of the magnetic susceptibility since a large range of values exists between the ferromagnetic state and the liquid state. In figures $8 \mathrm{a}$ and $8 \mathrm{~b}$, the different phase transitions are evidenced by the calculation of $\mathrm{d} \chi / \mathrm{d} T$ versus temperature. On the heating curve, one can see the ferromagnetic transition of a metastable phase called A1 which appears in rapid solidification due to cold-mold casting and which has a Curie temperature around $245^{\circ} \mathrm{C}$. Two transitions at higher temperatures (around $600^{\circ} \mathrm{C}$ ) could be associated with the dissolution of this phase and its transformation into the more stable phase $\mathrm{Nd}_{2} \mathrm{Fe}_{17}$. Two large peaks at high temperature correspond to melting and solidification. At lower temperatures, during the cooling of the sample, 3 peaks correspond to the ferromagnetic transitions of different phases : $\mathrm{Nd}_{2} \mathrm{Fe}_{17}\left(T_{\mathrm{c}}=54^{\circ} \mathrm{C}\right), \mathrm{Nd}_{5} \mathrm{Fe}_{17}\left(T_{\mathrm{c}}=230^{\circ} \mathrm{C}\right)$ and $\mathrm{Nd}_{2} \mathrm{Fe}_{14} \mathrm{~B}\left(T_{\mathrm{c}}=312^{\circ} \mathrm{C}\right)$. The presence of this latter phase is quite surprising since no boron was introduced in the alloy but can be understood if some boron has diffused from the BN crucible we used.

Figure $9 \mathrm{a}$ represents the microstructure of the top of the sample, as seen by back scattering electron microscopy. The morphology of the bottom (Fig. 9b) is drastically different. Magnetic analysis during the cooling of the sample and S.E.M. both confirm the separation of the eutectic in two domains : the bottom corresponds to an eutectic of $\mathrm{Nd}$ and $\mathrm{Nd}_{5} \mathrm{Fe}_{17}$ while the top corresponds to an eutectic of $\mathrm{Nd}$ and $\mathrm{Nd}_{2} \mathrm{Fe}_{17}$, with some $\mathrm{Nd}$ dendrites. We have checked that 


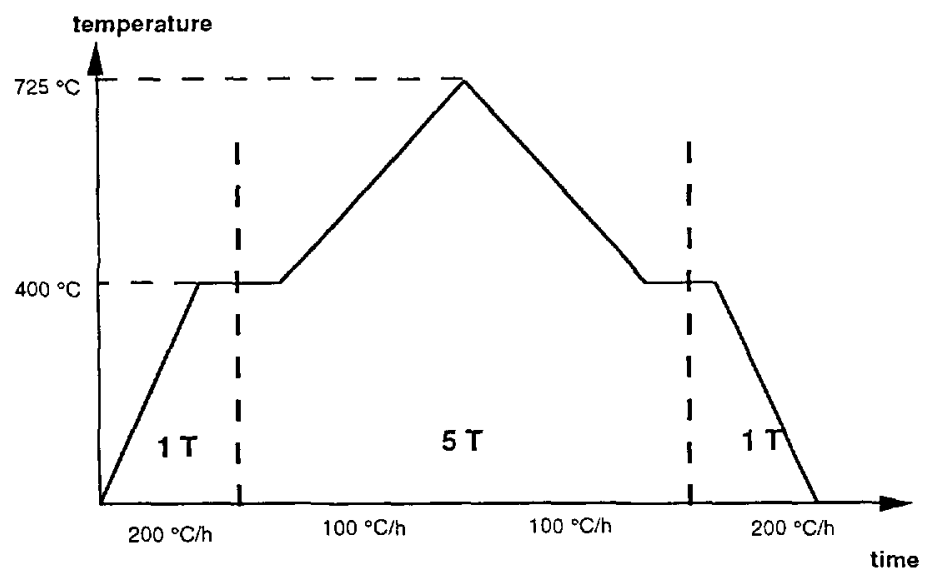

Fig. 7. - Thermal treatment applied to a $\mathrm{Nd}-\mathrm{Fe}$ eutectic alloy placed in the region of maximum downward magnetic force. Two different values of the magnetic field were used depending on the temperature since a very strong magnetic force exists at low temperature in the ferromagnetic state. The maximum temperature is about $40^{\circ} \mathrm{C}$ above the eutectic temperature.

no variation of the global composition was detected along the direction of the applied force. In contrast, a zero field solidified sample exhibits a homogeneous morphology where $\mathrm{Nd}, \mathrm{Nd}_{5} \mathrm{Fe}_{17}$, $\mathrm{Nd}_{2} \mathrm{Fe}_{17}$ are thoroughly randomly distributed (Fig. 10). The perfect separation in two domains with the application of a magnetic force (Fig. 9) suggests that just above the melting point or during solidification, nuclei of the different phases coexisting with a liquid phase allowing their migration were separated according to the amplitude of their magnetic susceptibility.

\section{Grain-oriented solidification.}

It is well knwon that particles of an anisotropic material immersed in a fluid may be oriented by a static magnetic field, providing there exists a shape anisotropy or a magnetic anisotropy giving a non zero mechanical momentum [11]. In the case of shape anisotropy, the anisotropic demagnetizing field gives a lower magnetic energy when the longest direction is aligned parallel with the magnetic field. In the case of magnetic susceptibility anisotropy, it is the axis of highest algebraïc magnetization aligned parallel with the field which gives the lowest energy. We have applied this phenomenon to the preparation of grain-oriented material by solidification in a magnetic field. To be successful, this orientation technique supposes that :

- solid crystallites coexist with a liquid phase ;

- magnetization anisotropy or shape anisotropy still exists at high temperature near the melting point ;

- this anisotropy and the applied field are high enough to counterbalance the thermal agitation $K_{\mathrm{B}} T$.

We have applied this technique to several materials, knowing or assuming that a magnetic anisotropy exists near their solidification point. In figure 11, we have plotted the magnetic susceptibility versus temperature of a sample of pure $\mathrm{Bi}$ first solidified in zero field. The lower curve corresponds to the heating and melting of the sample, while the upper curve corresponds 


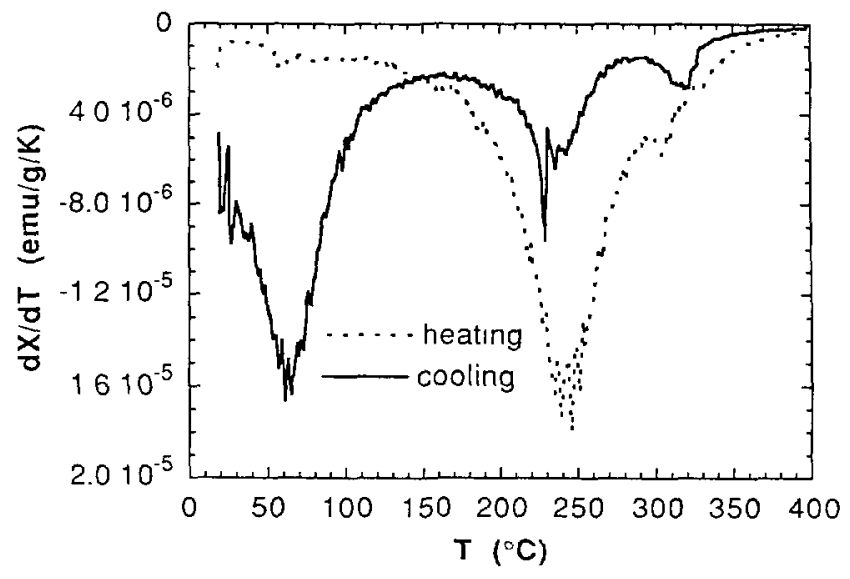

a)

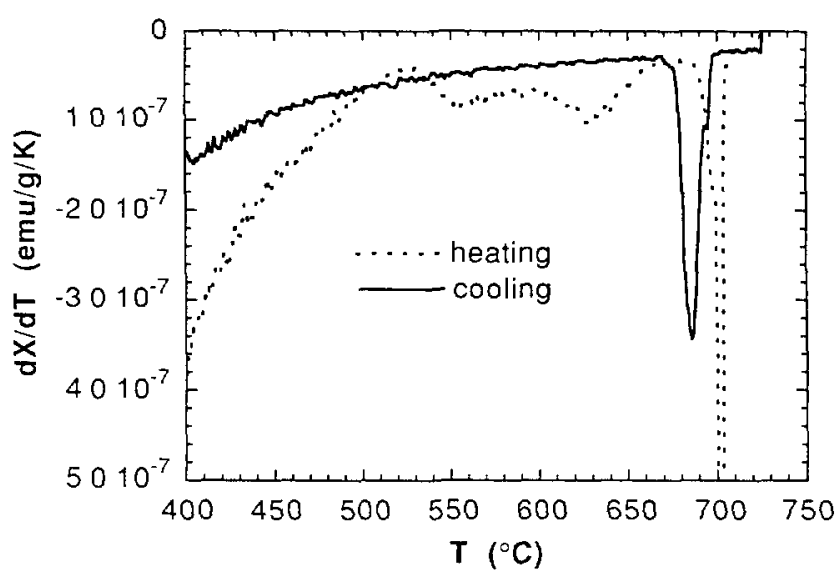

b)

Fig. 8. - Thermal derivative of the magnetic susceptibility versus temperature of a Nd-Fe eutectic alloy. The dotted line corresponds to the heating of a cold mold cast alloy. The full line corresponds to the cooling of the sample after melting and solidification in a magnetic force. A magnetic field of 1 tesla was applied below $400{ }^{\circ} \mathrm{C}$ (Fig. 8a) and 5 teslas above (Fig. 8b). The first peak in the heating curve is the ferromagnetic transition of a metastable phase $A 1\left(T_{c}=245^{\circ} \mathrm{C}\right)$. Two small peaks around $600^{\circ} \mathrm{C}$ could be associated to the dissolution of this phase. The sharp peaks at high temperature correspond to the melting and solidification of the alloy. The three peaks in the full line seen during the cooling below $400^{\circ} \mathrm{C}$ correspond to the ferromagnetic transitions of $\mathrm{Nd}_{2} \mathrm{Fe}_{14} \mathrm{~B}$ $\left(T_{\mathrm{c}}=312^{\circ} \mathrm{C}\right), \mathrm{Nd}_{5} \mathrm{Fe}_{17}\left(T_{\mathrm{c}}=230^{\circ} \mathrm{C}\right)$ and $\mathrm{Nd}_{2} \mathrm{Fe}_{17}\left(T_{\mathrm{c}}=54^{\circ} \mathrm{C}\right)$.

to the solidification and cooling in $5 \mathrm{~T}$. The large difference between the two curves is due to an alignment of solidifying crystallites with the $c$ axis of the hexagonal structure parallel to the applied field. The susceptibility at room temperature, $-1.04 \times 10^{-6} \mathrm{emu} / \mathrm{g}$ is the same as the known value of single crystals measured along the $c$ axis. X-ray diffraction patterns of cleavage plans have confirmed this alignment (Fig. 12). Similar results have also been observed with paramagnetic congruent melting alloys such as $\mathrm{CeNi}$ (Fig. 13) and $\mathrm{Dy}_{3} \mathrm{Al}_{2}$, confirming that during solidification, crystallites are free to rotate and align with their axis of major 

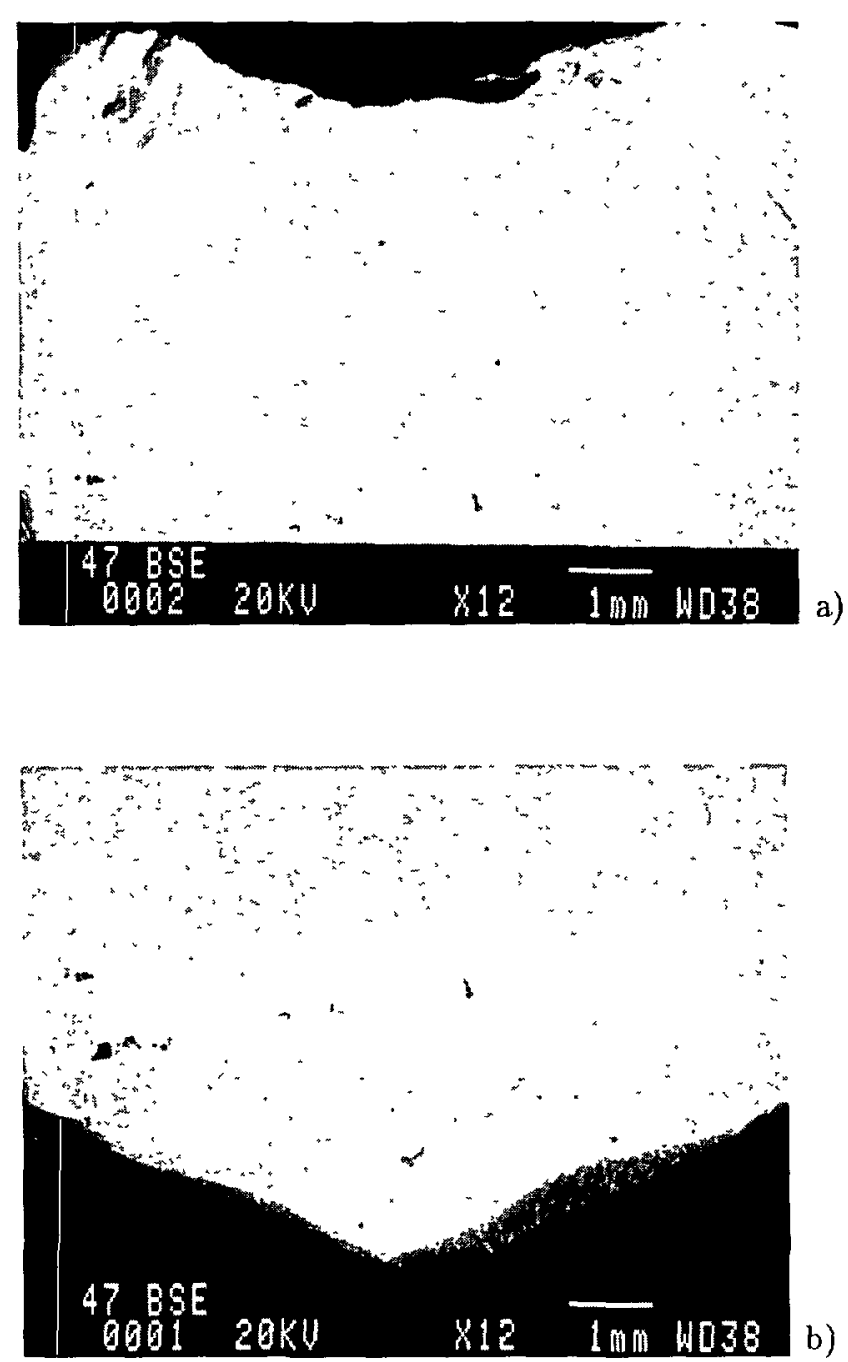

Fig. 9. - Back scattering electron microscopy of a polished face cut along the direction of the magnetic force applied during the solidification of a Nd-Fe eutectic alloy. The magnetic force has separated the alloy into two distinct domains, although the average composition is almost constant over all the sample. a) The top of the sample is an eutectic of $\mathrm{Nd}$ and $\mathrm{Nd}_{2} \mathrm{Fe}_{17}$ with dendrites of $\mathrm{Nd}$. b) The bottom of the sample is an eutectic of $\mathrm{Nd}$ and $\mathrm{Nd}_{5} \mathrm{Fe}_{17}$.

susceptibility parallel to the field.

This orientation effect has been also observed during the peritectic reaction of $\mathrm{YBa}_{2} \mathrm{Cu}_{3} \mathrm{O}_{7}$, leading to an enhancement of superconducting properties at low temperature [12]. To confirm that an anisotropy of the magnetic susceptibility still exists at high temperature, we have studied the alignment of $\mathrm{RBa}_{2} \mathrm{Cu}_{3} \mathrm{O}_{7}$ particles in liquid silver at $1020^{\circ} \mathrm{C}$, with $\mathrm{R}=\mathrm{Y}, \mathrm{Sm}, \mathrm{Eu}, \mathrm{Gd}$, $\mathrm{Dy}, \mathrm{Ho}, \mathrm{Er}$ and $\mathrm{Yb}$. We have measured at $4.2 \mathrm{~K}$ the magnetic susceptibility of those samples along the direction of the applied alignment field $H_{\mathrm{a}}$ and perpendicular to $H_{\mathrm{a}}$. Figure $14 \mathrm{a}$ is the curve measured on a cube taken from a sample with $\mathrm{R}=\mathrm{Er}$ prepared with $H_{\mathrm{a}}=7 \mathrm{~T}$. In 


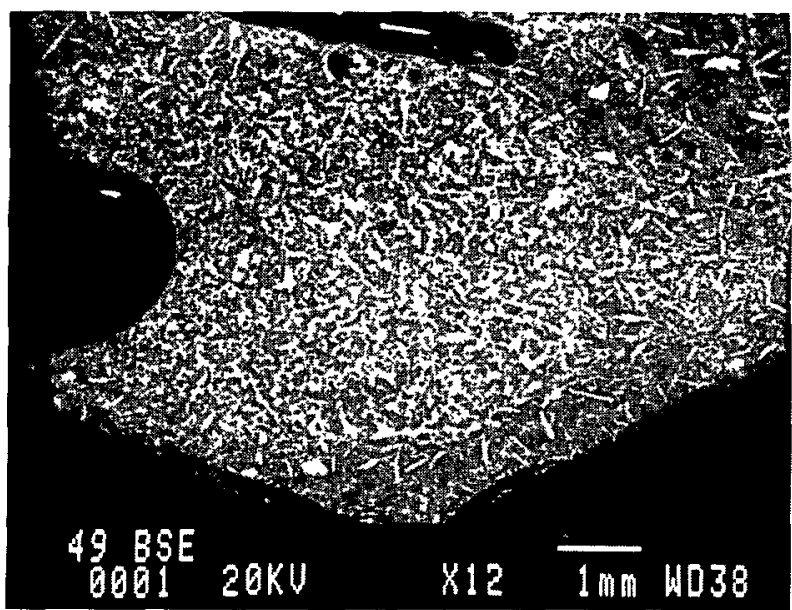

Fig. 10. - The morphology of a sample of Nd-Fe eutectic alloy prepared with no magnetic force, as seen by back scattering electron microscopy is quite different: We observed a random dispersion of $\mathrm{Nd}, \mathrm{Nd}_{5} \mathrm{Fe}_{17}$ and $\mathrm{Nd}_{2} \mathrm{Fe}_{17}$.

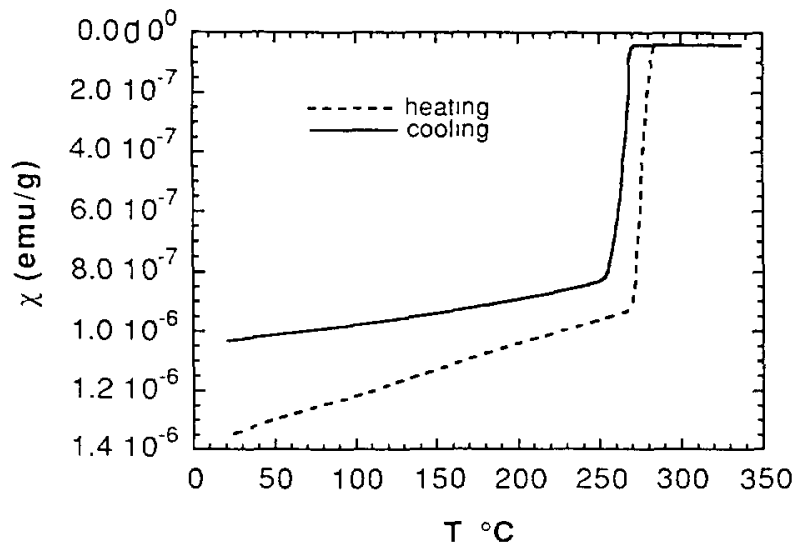

Fig. 11. - Magnetic susceptibility versus temperature of pure bismuth heated (dotted line) and solidified in a magnetic field (full line). A large variation of the susceptibility is measured between the solid and the liquid. The higher susceptibility measured after solidification is due to the alignment of solidifying crystallites in the magnetic field.

zero field (Fig. 14b) no anisotropy is observed. We define an alignment factor $r$ as the ratio of the width of the magnetization hysteresis measured at $4.2 \mathrm{~K}$ parallel and perpendicular to $H_{\mathrm{a}}$. In table II, the values measured on our sample as well as the direction of this orientation are compared to the values observed by other authors on powders oriented at room temperature in epoxy $[13,14]$. The orientation is governed by several terms : the magnetic anisotropy associated with the $\mathrm{CuO}$ planes, the magnetic anisotropy associated with the rare earth when it is substituted to $\mathrm{Y}$ and a shape effect that leads to an orientation with the applied field 

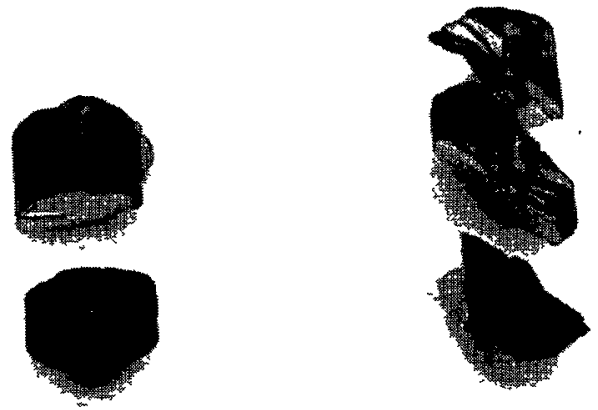

\section{$B=5 T$}

$\mathbf{B}=\mathbf{O} \mathbf{T}$

Fig. 12. - Cleavage along the ab plane of two samples of bismuth solidified with (Fig. 12a) and without magnetic field (Fig. 12b). In the field-solidified sample, the ab planes is perpendicular to the field applied during solidification, while it is not oriented without field (Fig. 12b).

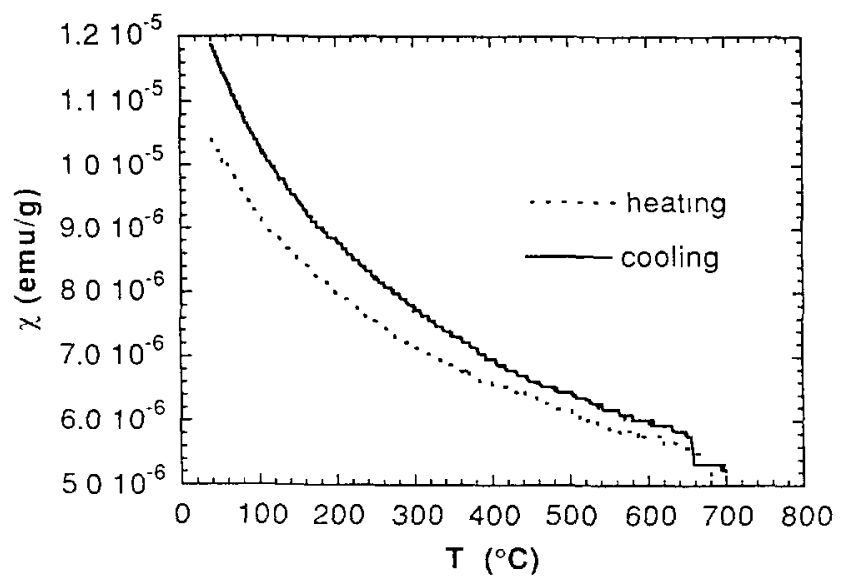

Fig. 13. - Magnetic susceptibility versus temperature of a CeNi sample. The dotted line corresponds to the heating of a zero field solidified sample. The higher susceptibility measured after solidification in 5 teslas (full line) is due to the align ment of crystallites with their axis of major susceptibility parallel to the applied field.

parallel to the ab planes.

One can see that :

- the $\mathrm{YBa}_{2} \mathrm{Cu}_{3} \mathrm{O}_{7}$ sample has been successfully aligned, confirming the existence at high temperature of a significant magnetic anisotropy associated with the $\mathrm{CuO}$ planes ;

- with $\mathrm{R}=\mathrm{Sm}$, Dy and Ho, the orientation at room temperature and high temperature 
corresponds to a magnetic anisotropy effect associated with both the $\mathrm{CuO}$ planes and the rare earth ;

- with $\mathrm{R}=\mathrm{Er}$ and $\mathrm{Yb}$, the orientation is due to the anisotropy of the rare earth but is almost counterbalanced by the $\mathrm{CuO}$ planes anisotropy in the case of $\mathrm{Yb}$ at high temperature ;

- with $\mathrm{R}=\mathrm{Eu}$, the orientation at room temperature corresponds to the anisotropy of the rare earth while the high temperature, the $\mathrm{CuO}$ planes anisotropy becomes predominant ;

- the sample with $\mathrm{R}=\mathrm{Gd}$ also exhibits a change in the orientation, from shape effect at room temperature to magnetic anisotropy effect at high temperature : this change can be understood since shape effect varies with $\chi^{2}$, i.e. with $T^{-2}$, whereas the magnetic anisotropy associated with the $\mathrm{CuO}$ planes should not vary rapidly with the temperature if we extrapolate the values measured between $100 \mathrm{~K}$ and $400 \mathrm{~K}$ by Miljak et al. [15].
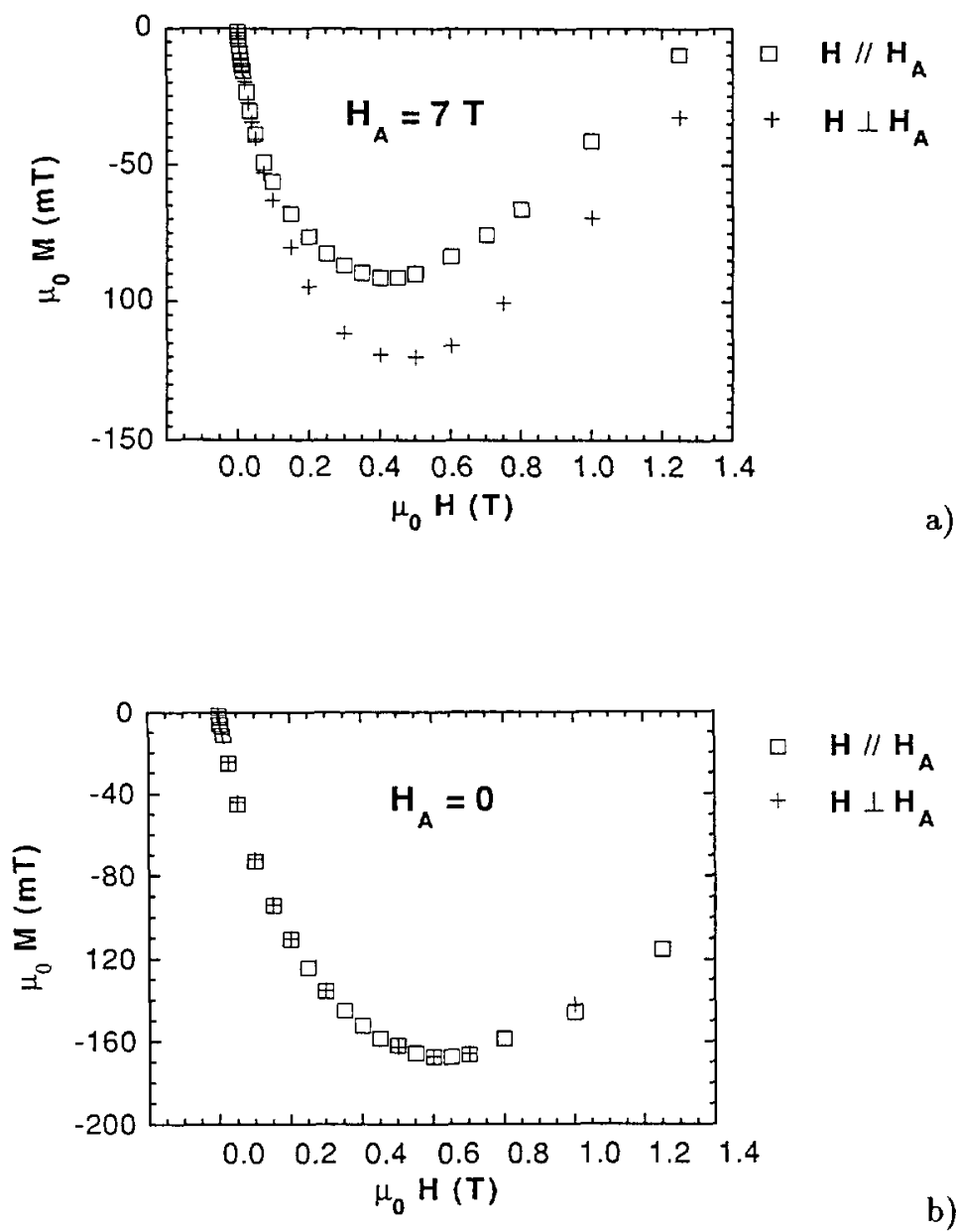

Fig. 14. - Magnetization versus field measured at $4.2 \mathrm{~K}$ on $2 \mathrm{~mm}$ side cubes cut from samples of $1 / 3$ $\mathrm{ErBa}_{2} \mathrm{Cu}_{3} \mathrm{O}_{7}+2 / 3 \mathrm{Ag}_{2} \mathrm{O}$ prepared with (Fig. 14a) and whithout (Fig. 14b) magnetic field. While no anisotropy is observed on a zero field solidified sample, the anisotropy measured on a sample solidified in a field of teslas corresponds to an alignment of the $c$ axis of the crystallites perpendicular to the applied field. 
Table II. - Orientation of $\mathrm{RBa}_{2} \mathrm{Cu}_{3} \mathrm{O}_{7}$ Crystallites in magnetic field at high temperature as compared to the orientation observed at room temperature by other authors.

\begin{tabular}{|c|c|c|c|c|}
\hline & Orientation at & $T=20^{\circ} \mathrm{C}$ & Orientation at & $T=1020^{\circ} \mathrm{C}$ \\
\hline & $c / H_{\mathrm{a}}$ & $r$ & $c / H_{\mathrm{a}}$ & $r$ \\
\hline $\mathrm{YBaCuO}$ & $/ /$ & $1.8[13]$ & $/ /$ & 1.4 \\
\hline $\mathrm{SmBaCuO}$ & $/ /$ & $3.5[13]$ & $/ /$ & 1.7 \\
\hline $\mathrm{EuBaCuO}$ & $\perp$ & $0.26[13]$ & $/ /$ & 1.4 \\
\hline $\mathrm{GdBaCuO}$ & $\perp$ & $\mathrm{nc}[14]$ & $/ /$ & 2.0 \\
\hline $\mathrm{DyBaCuO}$ & $/ /$ & $4.3[13]$ & $/ /$ & 1.3 \\
\hline $\mathrm{HoBaCuO}$ & $/ /$ & $5.3[13]$ & $/ /$ & 1.6 \\
\hline $\mathrm{ErBaCuO}$ & $\perp$ & $0.67[13]$ & $\perp$ & 0.71 \\
\hline $\mathrm{YbBaCuO}$ & $\perp$ & $0.30[13]$ & $\perp$ & 0.91 \\
\hline
\end{tabular}

Since promising results had been obtain in liquid silver with $\mathrm{R}=\mathrm{Y}$, we have studied the solidification of $\mathrm{YBa}_{2} \mathrm{Cu}_{3} \mathrm{O}_{7}$ in a magnetic field. Samples were prepared by grinding and compacting pellets of a mixture of $\mathrm{Y}_{2} \mathrm{BaCuO}_{5}, \mathrm{BaCuO}_{2}$ and $\mathrm{CuO}$. The pellets were submitted to the thermal treatment under magnetic field described in figure 15. X-ray diffraction, pole figures, scanning electron microscopy and magnetic measurements in the superconducting state confirm the orientation of the samples with the $c$ axis of the orthorhombic structure parallel to the applied field. Figure 16 shows platelets of $\mathrm{YBa}_{2} \mathrm{Cu}_{3} \mathrm{O}_{7}$ corresponding to the ab planes and aligned perpendicular to the field. In figure 17, we have measured the magnetic hysteresis in the superconducting state of two samples prepared with an without magnetic field. While no anisotropy in the superconducting state is measured in sample prepared without magnetic field, a large anisotropy can be seen between the direction parallel to the applied field $H_{\mathrm{a}}$ and a perpendicular one in a sample prepared with a magnetic field $H_{\mathrm{a}}=5 \mathrm{~T}$. As for rare earth samples oriented in liquid silver, the anisotropy in the superconducting state is related to the crystallographic orientation induced by the magnetic field during the solidification since the larger hysteresis corresponds to superconducting currents developed in the ab planes, i.e. when the measuring field is parallel to the $c$ direction which is the direction aligned by the applied field $H_{\mathrm{a}}$.

One can also see a large enhancement of the value of the magnetization of an oriented sample as compared to a disordered one (Fig. 17). In the first case, the alignment of the platelet-like crystals allows a good connection between the $a b$ planes and is favorable to reduce the weak links between two grains. As well as the enhancement of the magnetization along the direction of field-induced orientation, we have also observed high critical transport current density along a direction perpendicular to the field-induced orientation, i.e. along the $a b$ planes. In our best samples, current densities higher than $10000 \mathrm{~A} / \mathrm{cm}^{2}$ have been measured in $7 \mathrm{~T}$ at $77 \mathrm{~K}$.

\section{Effect of super heating on the orientation during solidification.}

In the search for the best process to be applied for a successful alignment of $\mathrm{YBa}_{2} \mathrm{Cu}_{3} \mathrm{O}_{7}$ grains during the peritectic reaction, we have studied the effect of the variation of the maximum 


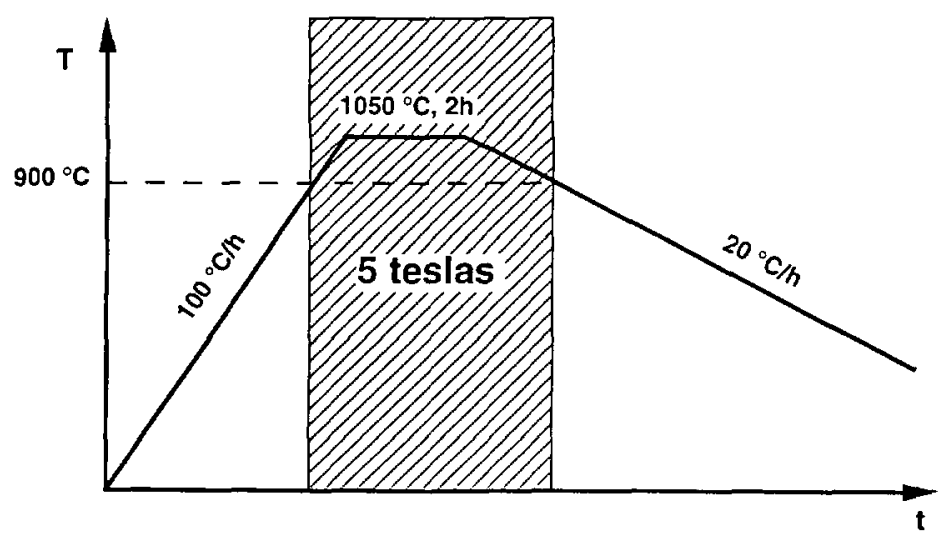

Fig. 15. - Magneto-thermal treatment applied to $\mathrm{Y}_{2} \mathrm{BaCuO}_{5}+3 \mathrm{BaCuO}_{2}+2 \mathrm{CuO}$ to obtain a grainoriented microstructure after solidification in a magnetic field.

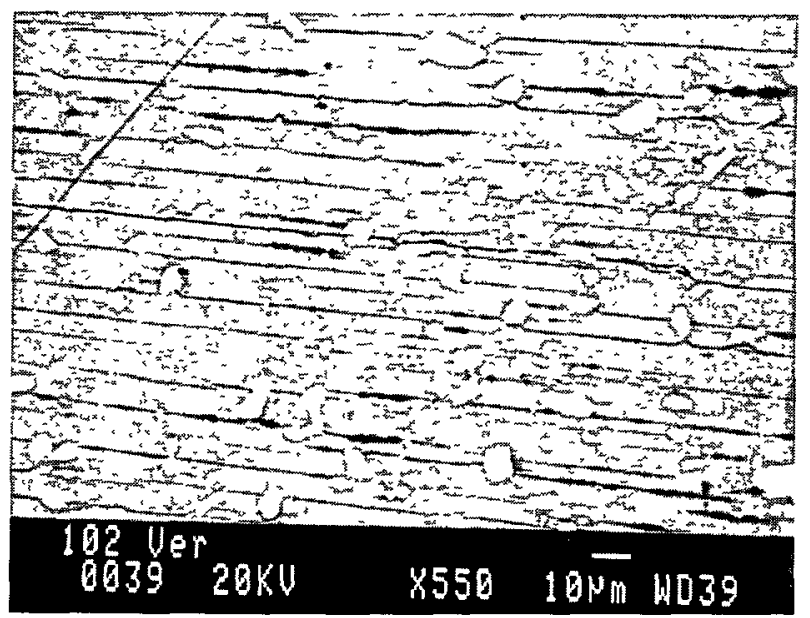

Fig. 16. - SEM microscopy of a polished face of field-solidified $\mathrm{YBa}_{2} \mathrm{Cu}_{3} \mathrm{O}_{7}$ sample. The face was cut parallel to the applied field. One can see the alignment of platelets which correspond to the $a b$ planes of the structure. The $a b$ planes are perpendicular to the magnetic field.

temperature $T_{M}$ reached during the thermal treatment under magnetic field. The alignment, as measured by the orientation factor $r$ defined earlier, is plotted as a function of $T_{M}$, from $1030{ }^{\circ} \mathrm{C}$ to $1070^{\circ} \mathrm{C}$ (Fig. 18). As we could expect, no or little orientation is obtained for low $T_{M}$, since the small amount of liquid phases due to incomplete peritectic reaction does not allow the free rotation of crystallites. More surprising is the fact that higher temperatures, well above the peritectic reaction temperature, lead to an incomplete orientation.

Similar features have been observed during the solidification under magnetic field of pure bismuth. In this case, we did not vary the maximum temperature but the cooling rate, i.e. the time elapsed in the liquid state. In figure 19, we present the results obtained for different colling rates. In each experiment we used the same sample, the temperature being rapidly 


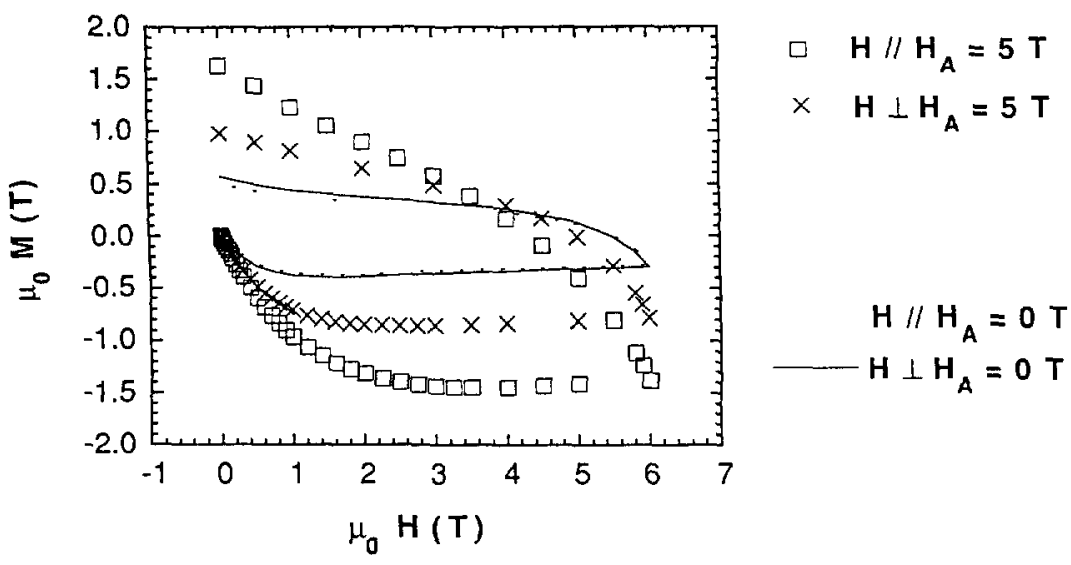

Fig. 17. - Magnetization versus field measured at $4.2 \mathrm{~K}$ of $\mathrm{YBa}_{2} \mathrm{Cu}_{3} \mathrm{O}_{7}$ samples prepared with and without magnetic field. No anisotropy is observed in the zero field solidified sample. When solidified in 5 teslas, the sample exhibits a larger magnetization and a strong anisotropy corresponding to an alignment of the $c$ axis of the crystallites parallel to the applied field.

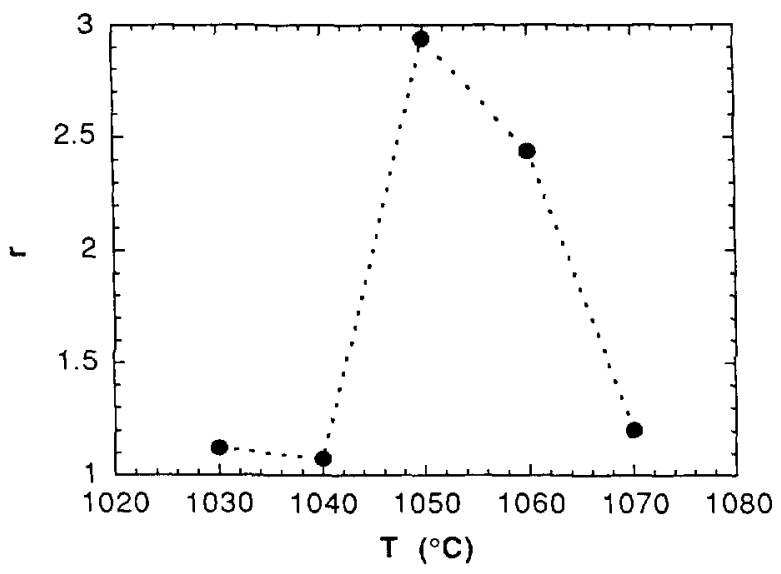

Fig. 18. - Anisotropy ratio of field solidified $\mathrm{YBa}_{2} \mathrm{Cu}_{3} \mathrm{O}_{7}$ samples as a function of the maximum temperature reached during the thermal treatment. The ratio is defined as the ratio of the width of the magnetization hysteresis measured at $4.2 \mathrm{~K}$ parallel and perpendicular to the field applied during solidification. A maximum is clearly seen a few degrees above the peritectic temperature. For lower temperatures the incomplete reaction leads to a non oriented sample. Misorientation observed for high maximum temperatures suggests that metastable germs of the $\mathrm{YBa}_{2} \mathrm{Cu}_{3} \mathrm{O}_{7}$ phase exist above the peritectic temperature and play an important role in the orientation during solidification. When too high temperatures are reached, those germs are dissolved leading to no orientation.

raised to $330{ }^{\circ} \mathrm{C}$, about $60^{\circ} \mathrm{C}$ aboce the melting point, and the cooling rate being varied from $650^{\circ} \mathrm{C} / \mathrm{h}$ to $25^{\circ} \mathrm{C} / \mathrm{h}$. The orientation was deduced from the susceptibility measured at room temperature along the direction of the field applied during the solidification. A $100 \%$ orientation corresponds to the susceptibility of a single crystal measured along the $c$ axis, while 
$0 \%$ indicates the susceptibility of a fully disordered polycrystalline sample. For low cooling rates, we observed supercooling and a tendency to give the susceptibility of a disordered sample. For high cooling rate, i.e. little time elapsed in the liquid state, we always obtained a perfectly oriented sample.

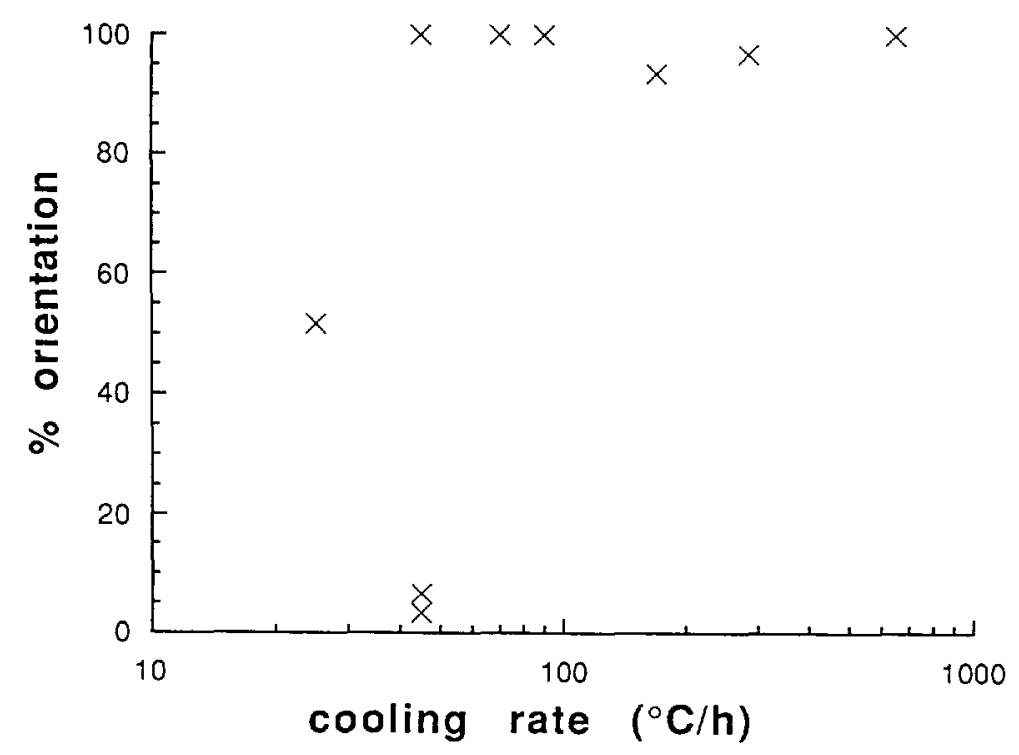

Fig. 19. - Observed orientation on, field solidified samples of bismuth as a function of the cooling rate of the furnace. A $100 \%$ orientation corresponds to a perfect alignment of the $c$ axis of the bismuth crystals parallel to the field applied during solidification, while $0 \%$ corresponds to an isotropic polycrystal. For high cooling rates, i.e. low time elapsed in the liquid state, a perfect orientation is obtained, while samples cooled at less than $50^{\circ} \mathrm{C} / \mathrm{h}$ are randomly oriented. These results are to be compared with those obtained with $\mathrm{YBa}_{2} \mathrm{Cu}_{3} \mathrm{O}_{7}$. metastable solid germs coexisting with the liquid are dissolved at high temperature or after a long time elapsed in the liquid state. These germs can no longer help for the orientation when too high temperatures or to small cooling rates are used.

This irreversible behavior of the liquid state may be seen in the view of the remaining of small solid nuclei superheated in the liquid state. Recently, Cahn [16] discussed the nature of the melting transition, reporting on the study by other authors of a succession of limits in the superheating of crystals. Indeed, in particular cases, superheating up to $480 \mathrm{~K}$ has been confirmed [17]. Our own results suggest that the liquid a few degrees above the melting point or the peritectic reaction is not in the same state that the same liquid at the same temperature but after an excursion at higher temperature. In the first case, solid particles are allowed to remain at high temperature, to align parallel with the applied field and to act as nuclei for the oriented solidification of the crystal. In the second case, heating the nuclei at higher temperatures or maintaining them a longer time at high temperature, may have fully dissolved them in the liquid, leading to no possible orientation in the liquid state and being responsible for the supercooling observed in bismuth by magnetic analysis. Moreover, such supercooling could prevent from the orientation of solidifying particles due to the catastrophic germination in the liquid. 


\section{Conclusions.}

We have performed an experimental and model study of the effects of a high static homogeneous or inhomogeneous magnetic field on material processing.

We have demonstrated that using the magnetic force that exists in magnetic field gradient numerous diamagnetic materials, in spite of their low susceptibility, can be maintained in zero gravity since high field apparatus are now available. This levitation is stable and allows a contactless preparation of material to avoid reactivity from a crucible.

Besides the suppression of buoyancy driven convection in simulated zero gravity, it is also possible to suppress, damp or enhance convection in liquids whose susceptibility varies with temperature. A much lower magnetic force than that used to obtain levitation is required to control this effect. The driving force of magnetic convection is independent of the electrical conductivity of the liquid.

We have appear during the solidification of a multiphased alloy. The magnetic force could then be used as a new tool to select, i.e. extract or sediment phases in a solidification process.

We have demonstrated that a large magnetic force is able to orientate solidifying crystallites in a peritectic reaction and in a congruent solidification, which leads to a textured material. This technique has led to a new process of high $\mathrm{T}_{\mathrm{c}}$ superconductors texturation.

We have observed the detrimental effect of superheating of the liquid on the magnetic field induced orientation during solidification. Our results suggest that metastable superheated solid nuclei of large size are present in the melting pot and play an important role in the induced orientation and that large superheating of the liquid destroy those nuclei.

\section{Acknowledgements.}

This work was supported by :

- Alcatel Alsthom Recherche

- Merlin Gerin

- Framatome

- Rhône-Poulenc

- Ministère de la Recherche et de l'Espace

- DRET

- PIRMAT (CNRS)

This paper is dedicated to the memory of Rammal Rammal who has been a fervent supporter of the development of this new research field.

\section{References}

[1] Vives Ch., J. Crystal Growth 76 (1986) 170.

[2] Savitsky E.M., Torchinova R.S., Turanov S.A., J. Crystal Growth 52 (1981) 519.

[3] Mikelson A.E., Karkin Ya.Kh., J. Crystal Growth 52 (1981) 524.

[4] Beaugnon E., Tournier R., Nature 349 (1991) 470.

[5] Beaugnon E., Tournier R., J. Phys. III France 1 (1991) 1423.

[6] Carruthers J.R., Wolfe R., J. Appl. Phys. 39 (1968) 5718.

[7] Braithwaite D., Beaugnon E., Tournier R., Nature 354 (1991) 134. 
[8] Landgraf F.J.G., Schneider G.S., Villas-boas V., Missel F.P., J. Less-Common Metals 163 (1990) 209.

[9] Cabral F.A.O., Gama S., J. Less-Common Metals 167 (1990) 31.

[10] Cabral F.A.O., Gama S., IEEE Trans. Mag. 26 (1990) 2622.

[11] Abritska M.YU., Vitole V.Kh., Karklin Ya.Kh., Mikelson A.E., Moshnyaga V.N., SEMINET S.I., Magnitnaya Gidiodinamica 3 (1976) 125.

[12] de Rango P., Lees M., Lejay P., Sulpice A., Tournier R., Ingold M., Germi P., Pernet M., Nature 349 (1991) 770.

[13] Ferreira J.M., Maple M.B., Zhou H., Make R.R., Lee B.W., Seaman C.L., Kuric M.W., Guertin R.P., J. Appl. Phys. A47 (1988) 105.

[14] Livingston J.D., Hart H.R., Wolf W.P., J. Appl. Phys. 64 (1988) 5806.

[15] Miljak M., Collin G., HaMzic A., J.M.M.M. 76-77 (1988) 609.

[16] CAHN R.W., Nature 342 (1989) 619.

[17] Rossouw C.J., Donnely S.E., Phys. Rev. Lett. 55 (1985) 2960. 